\title{
The Gluons in Particles
}

\section{Bin Liang}

College of Science, Chongqing University of Posts and Telecommunication, Chongqing, China

Email: liangbin@cqupt.edu.cn

How to cite this paper: Liang, B. (2018) The Gluons in Particles. Journal of Modern Physics, 9, 202-206.

https://doi.org/10.4236/jmp.2018.92013

Received: December 18, 2017

Accepted: January 19, 2018

Published: January 22, 2018

Copyright (C 2018 by author and Scientific Research Publishing Inc. This work is licensed under the Creative Commons Attribution International License (CC BY 4.0).

http://creativecommons.org/licenses/by/4.0/

\begin{abstract}
This article proves that the number of gluons is conserved in strong interaction and non-conserved in weak interaction by extending the idea that the quark is composed of gluons to other particles. It explains why the parity is non-conserved in weak interaction, why the fermions could be divided into the three generations, why the antiparticle of neutrino is itself and why the neutrino oscillation exists.
\end{abstract}

\section{Keywords}

Quark, Gluon, Strong and Weak Interaction, Parity, Neutrino and Its Oscillation

\section{Introduction}

In the paper "On Quarks and Gluons" [1] we have assumed that the gluons and anti-gluons constitute 6 quarks and 6 anti-quarks, respectively. The quarks are

$$
u=g_{a} g_{b}, d=g_{a} g_{c}, s=g_{a} g_{d}, c=g_{b} g_{c}, t=g_{b} g_{d}, b=g_{c} g_{d},
$$

the anti-quarks are

$$
\bar{u}=\bar{g}_{a} \bar{g}_{b}, \bar{d}=\bar{g}_{a} \bar{g}_{c}, \bar{s}=\bar{g}_{a} \bar{g}_{d}, \bar{c}=\bar{g}_{b} \bar{g}_{c}, \bar{t}=\bar{g}_{b} \bar{g}_{d}, \bar{b}=\bar{g}_{c} \bar{g}_{d},
$$

where the electric charges of gluons $g_{l}$ and anti-gluons $\bar{g}_{l}(l=a, b, c, d)$ are

$$
\begin{gathered}
Q_{a}=Q_{c}=Q_{d}=-1 / 6, Q_{b}=\frac{5}{6}, \\
\bar{Q}_{a}=\bar{Q}_{c}=\bar{Q}_{d}=\frac{1}{6}, \bar{Q}_{b}=-\frac{5}{6} .
\end{gathered}
$$

The spin of every gluon and anti-gluon is $1 / 4$, and their energy $\varepsilon_{l}$ obeys the inequality $\varepsilon_{a}<\varepsilon_{c}<\varepsilon_{d}<\varepsilon_{b}$. Thus, all of quarks and anti-quarks are fermions with spin $1 / 2$, and the charge of every quark or anti-quark equals to the sum of charges of gluons or anti-gluon in it.

It is needed to emphasize that, firstly, since all of gluons are fermions with 
spin1/4, the same gluons cannot constitute quark, but two different gluons in quark are commutable. Secondly, gluons have no mass, but quarks are massive. In fact, every particle with internal structures is massive, and vice versa.

We know that the mediators of nuclear force are the mesons $\pi$ and the mediators of weak interaction are the intermediate bosons. Since the intermediate bosons $W^{ \pm}$have the identical decay patterns with the mesons $\pi^{ \pm}$[2]:

$$
W^{ \pm}\left(\pi^{ \pm}\right) \rightarrow l^{ \pm}+v_{l}\left(\overline{v_{l}}\right)
$$

where $l$ can be electron $e$ or muon $\mu$, so the intermediate bosons $W^{ \pm}$ should also be composed of a quark and an anti-quark as same as the mesons $\pi^{ \pm}$, namely both strong and weak interaction should be related to gluons. If the idea that the quark is composed of gluons is extended to other particles, we will see that the number of gluons is conserved in strong interaction but non-conserved in weak interaction, the fermions could be divided into the three generations, the antiparticle of neutrino is itself and why the neutrino oscillation exists.

\section{The Gluon Number in Strong Interaction}

According to QCD, the mesons are composed of quark and anti-quark, and the baryons are composed of three quarks [3] [4]. So the strong interaction equation $\pi^{+}+n \rightleftharpoons p+\pi^{0}$ and $\pi^{-}+p \rightleftharpoons n+\pi^{0}$ can be written as

$$
\begin{aligned}
& \pi^{+}(u \bar{d})+n(\text { udd }) \rightarrow p(\text { uud })+\pi^{0}(u \bar{u}-d \bar{d}), \\
& \pi^{-}(\bar{u} d)+p(u u d) \rightarrow n(u d d)+\pi^{0}(u \bar{u}-d \bar{d}) .
\end{aligned}
$$

If we give the quark number +1 to each quark and the anti-quark number -1 to each anti-quark, the quark numbers on both sides of each equation above are equal:

$$
\begin{aligned}
& 2 u+1 d=2 u+1 d, \\
& 1 u+2 d=1 u+2 d .
\end{aligned}
$$

If we give the gluon number +1 to each gluon and the anti-gluon number -1 to each anti-gluon, respectively, using $u=g_{a} g_{b}, d=g_{a} g_{c}$ gives

$$
\begin{aligned}
& 3 g_{a}+2 g_{b}+1 g_{c}=3 g_{a}+2 g_{b}+1 g_{c}, \\
& 3 g_{a}+1 g_{b}+2 g_{c}=3 g_{a}+1 g_{b}+2 g_{c} .
\end{aligned}
$$

Obviously, the number of gluons is conserved in strong interaction, and this allows the charge, baryon number and parity to be conserved in strong interaction.

\section{The Gluon Number in Weak Interaction}

Is the number of gluons conserved in weak interaction? In the paper "On Quarks and Gluons" it is pointed out that the gluon and anti-gluon could annihilate into different particles in different interactional courses when they met. For example, $\pi^{ \pm} \rightarrow e^{ \pm}+v_{e}\left(\bar{v}_{e}\right)$ or $\mu^{ \pm}+v_{v}\left(\bar{v}_{\mu}\right)$. Using $\pi^{+}(u \bar{d}), \pi^{-}(\bar{u} d)$ and $u=g_{a} g_{b}$, $d=g_{a} g_{c}$, considering the spin and charge of the particles gives 


$$
\begin{aligned}
& \pi^{-}\left(\bar{g}_{a} \bar{g}_{b} g_{a} g_{c}\right) \rightarrow e^{-}\left(\bar{g}_{b} g_{c}\right)+\bar{v}_{e}\left(\bar{g}_{a} g_{a}\right), \\
& \pi^{+}\left(g_{a} g_{b} \bar{g}_{a} \bar{g}_{c}\right) \rightarrow e^{+}\left(g_{b} \bar{g}_{c}\right)+v_{e}\left(\bar{g}_{a} g_{a}\right), \\
& \pi^{-}\left(\bar{g}_{a} \bar{g}_{b} g_{a} g_{c}\right) \rightarrow \mu^{-}\left(\bar{g}_{b} g_{c}\right)+\bar{v}_{\mu}\left(\bar{g}_{a} g_{a}\right), \\
& \pi^{+}\left(g_{a} g_{b} \bar{g}_{a} \bar{g}_{c}\right) \rightarrow \mu^{+}\left(g_{b} \bar{g}_{c}\right)+v_{\mu}\left(\bar{g}_{a} g_{a}\right),
\end{aligned}
$$

As said previously, since the intermediate bosons $W^{ \pm}$have the identical decay patterns with the mesons $\pi^{ \pm}$:

$$
W^{ \pm}=e^{ \pm}+v_{e}\left(\bar{v}_{e}\right),
$$

so there should be $W^{+}(u \bar{d}), W^{-}(\bar{u} d)$. Thus the two other decay patterns of the intermediate bosons $W^{ \pm}$can be written as

$$
\begin{aligned}
& W^{-}\left(\bar{g}_{a} \bar{g}_{b} g_{a} g_{c}\right) \rightarrow \tau^{-}\left(\bar{g}_{b} g_{c}\right)+\bar{v}_{\tau}\left(\bar{g}_{a} g_{a}\right) \\
& W^{+}\left(g_{a} g_{b} \bar{g}_{a} \bar{g}_{c}\right) \rightarrow \tau^{+}\left(g_{b} \bar{g}_{c}\right)+v_{\tau}\left(\bar{g}_{a} g_{a}\right) .
\end{aligned}
$$

Obviously, the gluon number is conserved in every equation above. But, if the decay $K^{+} \rightarrow \pi^{+}+\pi^{0}$ and $\Sigma^{+} \rightarrow n+\pi^{+}$of strange particles are written as

$$
\begin{aligned}
& K^{+}(u \bar{s}) \rightarrow \pi^{+}(u \bar{d})+\pi^{0}(u \bar{u}-d \bar{d}), \\
& \Sigma^{+}(u u s) \rightarrow n(u d d)+\pi^{+}(u \bar{d}),
\end{aligned}
$$

or

$$
\begin{aligned}
& K^{+}\left(g_{a} g_{b} \bar{g}_{a} \bar{g}_{d}\right) \rightarrow \pi^{+}\left(g_{a} g_{b} \bar{g}_{a} \bar{g}_{c}\right)+\pi^{0}\left(g_{a} g_{b} \bar{g}_{a} \bar{g}_{b}-g_{a} g_{c} \bar{g}_{a} \bar{g}_{c}\right), \\
& \Sigma^{+}\left(g_{a} g_{b} g_{a} g_{b} g_{a} g_{d}\right) \rightarrow n\left(g_{a} g_{b} g_{a} g_{c} g_{a} g_{c}\right)+\pi^{+}\left(g_{a} g_{b} \bar{g}_{a} \bar{g}_{c}\right),
\end{aligned}
$$

both the quark number and gluon number are non-conserved, this is because of the existence of the decay $g_{d} \rightarrow g_{c}$ and $\bar{g}_{d} \rightarrow \bar{g}_{c}$. So, the reason why the parity is non-conserved in weak interaction is the gluon number is non-conserved.

\section{The Formation of Three Generations of Fermions}

From Equations ((9) and (11)) we see that the three neutrinos and their antiparticles are composed of gluon $g_{a}$ and anti-gluon $\bar{g}_{a}$, and the three leptons are composed of $\bar{g}_{b}, g_{c}$. How should this be explained?

As pointed out that in the paper "On Quarks and Gluons", the binding energy $u_{I l^{\prime}}$ of two spins $s_{l}, s_{l^{\prime}}$ has three values according to the Ising model:

$$
u_{I l^{\prime}}=-u_{f} \sum_{\left(I I^{\prime}\right)} s_{l} s_{I^{\prime}}=-u_{f}\left(5-2 N_{+}\right)= \begin{cases}-5 u_{f}, & N_{+}=0 \\ -3 u_{f}, & N_{+}=1 \\ -u_{f}, & N_{+}=2\end{cases}
$$

Therefore, the two gluons $g_{a}, \bar{g}_{a}$ can and can only constitute the three neutrinos in the three different ways, in other words, the three neutrinos are just the three states of the same particle, and the neutrinos antiparticle is itself. And since the energy spectrum of lepton in Equations ((9) and (11)) is continuous and tends to be maximal, and the energy spectrum of corresponding neutrino is discrete and tends to be minimal, so the neutrinos can be only composed of the gluons $g_{a}, \bar{g}_{a}$ of minimal energy rather than the gluons of higher energy. 
Similarly, since the gluon number is conserved in Equations (9) and (11), the three leptons and their antiparticles can be only composed of gluons $\bar{g}_{b}, g_{c}$ and $g_{b}, \bar{g}_{c}$ in the three different ways, respectively.

As shown in Equation (5), the six quarks have only two values of charge. Thus, the six quarks, three leptons and three corresponding neutrinos constitute the three generations of fermions as usually said:

$$
\begin{aligned}
& u, d, e, v_{e}, \\
& c, s, \mu, v_{\mu}, \\
& t, b, \tau, v_{\tau} .
\end{aligned}
$$

\section{The Reason of Neutrino Oscillation}

The static mass of neutrino can be written as (light speed $c=1$ )

$$
m=2 \varepsilon_{g}-V,
$$

where $\varepsilon_{g}$ is the energy of single gluon, and $V=-u_{l l^{\prime}}>0$ is the binding energy of two gluons. We know that the mass of three neutrinos $v_{e}, v_{\mu}, v_{\tau}$ is

$$
m_{v_{e}}<m_{v_{\mu}}<m_{v_{\tau}} .
$$

So their binding energy is

$$
V_{v_{e}}>V_{v_{\mu}}>V_{v_{\tau}}>0
$$

As said previously, the three neutrinos are the three states of the same particle. Since the smaller the binding energy is, the state of particle is more unstable, the neutrino state of smaller binding energy is going to decay into the state of greater binding energy, and this is the neutrino oscillation:

$$
\begin{aligned}
& v_{\tau} \rightarrow v_{\mu}, v_{e} \\
& v_{\mu} \rightarrow v_{e} .
\end{aligned}
$$

This is consistent with the experiments [5]-[10].

Using the method from Equations (16) to (19) we can also explain why there are only the decays of $\tau \rightarrow \mu, \tau \rightarrow e$ and $\mu \rightarrow e$ rather than the reversed decays:

$$
\begin{aligned}
& \tau^{ \pm} \rightarrow \mu^{ \pm}\left(e^{ \pm}\right)+\left\{\begin{array}{l}
\bar{v}_{\tau}+v_{\mu}\left(v_{e}\right), \\
v_{\tau}+\bar{v}_{\mu}\left(\bar{v}_{e}\right),
\end{array}\right. \\
& \mu^{ \pm} \rightarrow e^{ \pm}+\left\{\begin{array}{l}
\bar{v}_{\mu}+v_{e}, \\
v_{\mu}+\bar{v}_{e} .
\end{array}\right.
\end{aligned}
$$

\section{References}

[1] Liang, B. (2015) Journal of Modern Physics, 6, 982-989. https://doi.org/10.4236/jmp.2015.67102

[2] Lee, T.D. (1988) Particle Physics and Introduction to Field Theory. Harwood Acad., London, Chapter 21.

[3] Gell-Mann, M. (1962) Physical Review, 125, 1067. 
[4] Zweig, G. (1964) CERN-TH-412, 401.

[5] Ashine, Y., et al. (Super-K Collaboration) (2005) Physical Review D, D71, 112005.

[6] Michael, D., et al. (MINOS Collaboration) (2006) Physical Review Letters, 97, 191801. https://doi.org/10.1103/PhysRevLett.97.191801

[7] Adamson, P., et al. (MINOS Collaboration) (2008) Physical Review Letters, 101, 131802. https://doi.org/10.1103/PhysRevLett.101.131802

[8] Abe, S., et al. (KamLand Collaboration) (2008) Physical Review Letters, 100, 221803. https://doi.org/10.1103/PhysRevLett.100.221803

[9] Beringer, J., et al. (PDG Collaboration) (2012) Physical Review D, D86, 010001.

[10] An, F.P., et al. (Daya Bay Collaboration) (2012) Physical Review Letters, 108, 171803. https://doi.org/10.1103/PhysRevLett.108.171803 